\title{
Exponential of a Matrix, a Nonlinear Problem and Quantum Gates
}

\author{
Willi-Hans Steeb ${ }^{\dagger}$ and Yorick Hardy* \\ $\dagger$ International School for Scientific Computing, \\ University of Johannesburg, Auckland Park 2006, South Africa, \\ e-mail: steebwilli@gmail.com \\ * Department of Mathematical Sciences, \\ University of South Africa, Johannesburg, South Africa, \\ e-mail: hardyy@unisa.ac.za
}

\begin{abstract}
We describe solutions of the matrix equation $\exp \left(z\left(A-I_{n}\right)\right)=A$, where $z \in \mathbb{C}$. Applications in quantum computing are given. Both normal and nonnormal matrices are studied. For normal matrices, the Lambert W-function plays a central role.
\end{abstract}

\section{Introduction}

The motivation of this paper comes from the following observation. Let $\sigma_{1}, \sigma_{2}, \sigma_{3}$ be the Pauli spin matrices

$$
\sigma_{1}=\left(\begin{array}{cc}
0 & 1 \\
1 & 0
\end{array}\right), \quad \sigma_{2}=\left(\begin{array}{cc}
0 & -i \\
i & 0
\end{array}\right), \quad \sigma_{3}=\left(\begin{array}{cc}
1 & 0 \\
0 & -1
\end{array}\right)
$$

and $\sigma_{0} \equiv I_{2}$ be the $2 \times 2$ identity matrix, then

$$
\exp \left(-\frac{1}{2} i \pi\left(\sigma_{j}-I_{2}\right)\right) \equiv \sigma_{j}, \quad j=0,1,2,3
$$

These identities play a role in quantum theory [1]. An extension is

$\exp \left(-\frac{1}{2} i \pi\left(\sigma_{j_{1}} \otimes \sigma_{j_{2}} \otimes \cdots \otimes \sigma_{j_{n}}-I_{2^{n}}\right)\right) \equiv \sigma_{j_{1}} \otimes \sigma_{j_{2}} \otimes \cdots \otimes \sigma_{j_{n}}, \quad j_{k}=0,1,2,3$

where the $2^{n} \times 2^{n}$ matrices $\sigma_{j_{1}} \otimes \sigma_{j_{2}} \otimes \cdots \otimes \sigma_{j_{n}}$ are elements of the Pauli group. Thus we ask the question: find all $n \times n$ matrices $A$ over $\mathbb{C}$ and $z \in \mathbb{C}$ such that

$$
e^{z\left(A-I_{n}\right)}=A
$$


Equation (1) can also be written in the form $e^{z A}=e^{z} A$. The trivial solution is

given by $A=I_{n}$ with $z$ arbitrary. Since $\operatorname{det}\left(e^{M}\right) \equiv e^{\operatorname{tr}(M)}$ for any $n \times n$ matrix $M$ over $\mathbb{C}$ we obtain

$$
\operatorname{det}\left(e^{z\left(A-I_{n}\right)}\right)=e^{\operatorname{tr}\left(z\left(A-I_{n}\right)\right)}=e^{z(\operatorname{tr}(A)-n)}=\operatorname{det}(A)
$$

Since $\exp (z(\operatorname{tr}(A)-n))$ is nonzero we can conclude that $A$ must be invertible. Note that if $A$ and $B$ are similar matrices, then $B$ satisfies (1) when $A$ satisfies (1) and vice versa. An important special case for a solution of equation (1) can be given at once. Let $B$ be an $n \times n$ matrix with $B^{2}=I_{n}$. Then

$$
\exp \left(\frac{1}{2}(2 k+1) i \pi\left(B-I_{n}\right)\right) \equiv B \quad \forall k \in \mathbb{Z}
$$

The proof is based on the identity $(z \in \mathbb{C})$

$$
e^{z B} \equiv \cosh (z) I_{n}+\sinh (z) B
$$

for any $n \times n$ matrix $B$ with $B^{2}=I_{n}$. Setting $z=i \pi / 2+i k \pi$ we obtain the identity (2) utilizing that $\sin (\pi / 2+k \pi)=(-1)^{k}$ and $\cos (\pi / 2+k \pi)=0$. No other solution exists when $B^{2}=I_{n}$.

We investigate first normal matrices and then nonnormal matrices. Finally a number of applications are provided.

\section{Solutions for Normal Matrices}

We solve the problem under the assumption that $A$ is a normal matrix, i.e. $A A^{*}=$ $A^{*} A$. Let $U$ be the unitary matrix that diagonalizes $A$, i.e.

$$
U^{-1} A U=D \equiv \operatorname{diag}\left(\lambda_{1}, \ldots, \lambda_{n}\right)
$$

where $\lambda_{j}$ are the eigenvalues of $A$. Then from $e^{z\left(A-I_{n}\right)}=A$ we find

$$
U^{-1} e^{z\left(A-I_{n}\right)} U=U^{-1} A U \quad \Rightarrow \quad e^{z U^{-1}\left(A-I_{n}\right) U}=U^{-1} A U
$$

Consequently $e^{z\left(D-I_{n}\right)}=D$ and

$$
e^{z\left(\lambda_{j}-1\right)}=\lambda_{j}, \quad j=1, \ldots, n
$$

or equivalently

$$
e^{-z}=\lambda_{j} e^{-z \lambda_{j}}, \quad j=1, \ldots, n
$$


Clearly $z=0$ gives $A=I_{n}$. However, $A=I_{n}$ does not constrain $z$. In the following we restrict our discussion to the case $z \neq 0$ and $A \neq I_{n}$.

The solution of $e^{-z}=\lambda e^{-z \lambda}$ can be given as

$$
\lambda=-\frac{1}{z} W\left(-z e^{-z}\right)
$$

where $W$ is (any branch of) the Lambert $W$-function [2, 3, 4]. The Lambert $W$-function is defined by $z=W(z) \exp (W(z))$ with the properties

$$
W(0)=0, \quad W(e)=1, \quad W(-\pi / 2)=i \pi / 2 .
$$

Thus for any $z \in \mathbb{C}$ we can construct a normal matrix $A$ satisfying (1) using the Lambert $W$-function. In particular, consider $z=-i \omega t$, where $\omega$ is the frequency and $t$ the time. Then

$$
\lambda=\frac{1}{i \omega t} W\left(i \omega t e^{i \omega t}\right)
$$

With $\omega=\pi / 2$ we have $e^{i \omega t}=i$ and hence $\lambda=1$ since the Lambert $W$-function satisfies $W(-\pi / 2)=i \pi / 2$. The Lambert $W$-function for matrices has been studied by Higham [5] and Corliss et al [6].

Now we consider the relationship between eigenvalues of $A$. Since $e^{-z}=\lambda_{j} e^{-z \lambda_{j}}$ for all $j \in\{1, \ldots, n\}$. If $\lambda_{j}=1$ then the $j$-th equation is satisfied identically. If $\lambda_{j} \neq 1$ then there must exist $k_{j} \in \mathbb{Z}$ such that

$$
z=\frac{\ln \lambda_{j}+2 \pi k_{j} i}{\lambda_{j}-1}
$$

Suppose there exists $\lambda_{p} \neq 1$ with $\lambda_{p} \neq \lambda_{j}$, then we also have

$$
z=\frac{\ln \lambda_{p}+2 \pi k_{p} i}{\lambda_{p}-1}
$$

for some $k_{p} \in \mathbb{Z}$. It follows that

$$
k_{p}=\frac{1}{2 \pi i}\left[\frac{\lambda_{p}-1}{\lambda_{j}-1}\left(\ln \lambda_{j}+2 \pi k_{j} i\right)-\ln \lambda_{p}\right] \in \mathbb{Z} .
$$

We have the following cases:

1. $A=I_{n}, z \in \mathbb{C}$ 
2. $A$ has $r \neq 0$ distinct eigenvalues $\lambda_{1} \neq 1, \lambda_{2} \neq 1, \ldots, \lambda_{r} \neq 1$;

$$
z=\left(\ln \lambda_{1}+2 \pi k i\right) /\left(\lambda_{1}-1\right)
$$

for some $k \in \mathbb{Z}$ and for all $j \in\{1, \ldots, r\}$

$$
\frac{1}{2 \pi i}\left[\frac{\lambda_{j}-1}{\lambda_{1}-1}\left(\ln \lambda_{1}+2 \pi k i\right)-\ln \lambda_{j}\right] \in \mathbb{Z}
$$

and any remaining eigenvalues are 1.

Thus $A$ and $z$ satisfy (1) if and only if one of the above cases hold.

\section{Solutions for Nonnormal Matrices}

Note that also some nonnormal matrices $N$ can satisfy the condition that $N^{2}=I_{n}$, so that the solution (2) holds. Consider for example

$$
N=\left(\begin{array}{cc}
1 & \epsilon \\
0 & -1
\end{array}\right) \equiv \sigma_{3}+\left(\begin{array}{ll}
0 & \epsilon \\
0 & 0
\end{array}\right)
$$

with $\epsilon \neq 0$. Thus $N^{*} N \neq N N^{*}$. Another example is the matrix

$$
M=\left(\begin{array}{ccc}
1 & 0 & \epsilon \\
0 & -1 & 0 \\
0 & 0 & -1
\end{array}\right)
$$

with $\epsilon \neq 0$.

In general, if $A$ is a fixed $n \times n$ matrix, let $\left\{I_{n}, A, A^{2}, \ldots, A^{r}\right\}$ be the largest linearly independent set constructed from powers of $A$ (by the Cayley-Hamilton theorem $r \leq n-1)$ then there exists $c_{j}(z)(j=0,1, \ldots, r)$ such that

$$
\sum_{j=0}^{r} c_{j}(z) A^{j}=e^{z A}=e^{z} A .
$$

Thus any solutions $z$ satisfy

$$
c_{j}(z)=0, \quad j=0,2,3, \ldots, n \quad c_{1}(z)=e^{z} .
$$

The case $N^{2}=I_{n}$ above follows as a special case when $r=1$.

Similar to the normal matrices case in the previous section, the eigenvalues of $A$ obey the relations below. However, in these cases additional constraints on $U$ are necessary.

Assume $A$ satisfies (1), then we have the following cases: 
1. $A=I_{n}+U, z \in \mathbb{C}$; for some strictly upper triangular matrix $U \neq 0$

2. $A$ has $r \neq 0$ distinct eigenvalues $\lambda_{1} \neq 1, \lambda_{2} \neq 1, \ldots, \lambda_{r} \neq 1$;

$$
z=\left(\ln \lambda_{1}+2 \pi k i\right) /\left(\lambda_{1}-1\right)
$$

for some $k \in \mathbb{Z}$ and for all $j \in\{1, \ldots, r\}$

$$
\frac{1}{2 \pi i}\left[\frac{\lambda_{j}-1}{\lambda_{1}-1}\left(\ln \lambda_{1}+2 \pi k i\right)-\ln \lambda_{j}\right] \in \mathbb{Z}
$$

and any remaining eigenvalues are 1.

Thus if $A$ and $z$ satisfy (1) then one of the above cases hold.

\section{Applications}

Let $\hat{H}$ be the Hamilton operator acting in a finite dimensional Hilbert space $\mathbb{C}^{n}$. Thus $\hat{H}$ would be an $n \times n$ hermitian matrix. The solution of the Schrödinger equation is given by

$$
|\psi(t)\rangle=\exp (-i \hat{H} t / \hbar)|\psi(0)\rangle .
$$

Thus if $\hat{H}=\hbar \omega K$ with $K^{2}=I_{n}$ we have

$$
\exp (-i \omega t K)=\cos (\omega t) I_{n}-i \sin (\omega t) K
$$

where we utilized that $\cosh (-i \omega t) \equiv \cos (\omega t), \sinh (-i \omega t) \equiv-i \sin (\omega t)$. Thus to satisfy the equation $e^{z A}=e^{z} A$ we have to set $\omega t=\pi / 2$. Then we obtain

$$
\exp \left(-i \frac{\pi}{2} K\right)=e^{-i \pi / 2} K
$$

with $e^{-i \pi / 2}=-i$.

As an example consider the triple spin Hamilton operator (Steeb [7])

$$
\hat{H}=\hbar \omega\left(\sigma_{1} \otimes \sigma_{3} \otimes \sigma_{2}\right) .
$$

Since $\left(\sigma_{1} \otimes \sigma_{3} \otimes \sigma_{2}\right)^{2}=I_{2} \otimes I_{2} \otimes I_{2}$ we obtain

$$
e^{-i \hat{H} t / \hbar}=e^{-i \omega t\left(\sigma_{1} \otimes \sigma_{3} \otimes \sigma_{2}\right)}=I_{8} \cos (\omega t)-i \sin (\omega t)\left(\sigma_{1} \otimes \sigma_{3} \otimes \sigma_{2}\right) .
$$

If $\omega t=\pi / 2$, then $\cos (\pi / 2)=0, \sin (\pi / 2)=1$ and we obtain

$$
\exp \left(-i \frac{\pi}{2}\left(\sigma_{1} \otimes \sigma_{3} \otimes \sigma_{2}\right)\right)=-i \sigma_{1} \otimes \sigma_{3} \otimes \sigma_{2}
$$


where $e^{-i \pi / 2}=-i$.

Many quantum gates $\left([8,[9,10,11])\right.$ such as the Hadamard gate $U_{H}$ and the CNOT-gate

$$
U_{H}=\frac{1}{\sqrt{2}}\left(\begin{array}{cc}
1 & 1 \\
1 & -1
\end{array}\right), \quad U_{C N O T}=\left(\begin{array}{llll}
1 & 0 & 0 & 0 \\
0 & 1 & 0 & 0 \\
0 & 0 & 0 & 1 \\
0 & 0 & 1 & 0
\end{array}\right)
$$

and Swap gate

$$
U_{\text {swap }}=\left(\begin{array}{cccc}
1 & 0 & 0 & 0 \\
0 & 0 & 1 & 0 \\
0 & 1 & 0 & 0 \\
0 & 0 & 0 & 1
\end{array}\right)
$$

satisfy the condition that the square is the identity matrix.

If the $n \times n$ matrices $X$ and $Y$ satisfy $X^{2}=I_{n}, Y^{2}=I_{n}$, then $X \otimes Y$ and $X \oplus Y$ satisfy $(X \otimes Y)^{2}=I_{n^{2}}$ and $(X \oplus Y)^{2}=I_{2 n}$, where $\oplus$ denotes the direct sum. An application would be the Pauli spin matrices, for example $\sigma_{1} \otimes \sigma_{2}, \sigma_{1} \oplus \sigma_{2}$ or $\sigma_{1} \otimes \sigma_{3} \otimes \sigma_{2}, \sigma_{1} \oplus \sigma_{3} \oplus \sigma_{2}$. The elements $\sigma_{1} \otimes \sigma_{2}, \sigma_{1} \otimes \sigma_{3} \otimes \sigma_{2}$ are elements of the Pauli group. The $n$-qubit Pauli group $\mathcal{P}_{n}$ is defined by

$$
\mathcal{P}_{n}:=\left\{I_{2}, \sigma_{1}, \sigma_{2}, \sigma_{3}\right\}^{\otimes n} \otimes\{ \pm 1, \pm i\}
$$

The $n$-qubit Pauli group $\mathcal{P}_{n}$ is of order $4^{n+1}$.

\section{Conclusion}

We solved the matrix equation $\exp (z A)=\exp (z) A$ for normal matrices. We have also shown that solutions for nonnormal matrices exist.

\section{Acknowledgment}

The authors are supported by the National Research Foundation (NRF), South Africa. This work is based upon research supported by the National Research Foundation. Any opinion, findings and conclusions or recommendations expressed in this material are those of the author(s) and therefore the NRF do not accept any liability in regard thereto. 


\section{References}

[1] G. 't Hooft, "Quantummechanical behaviour in a deterministic model", Found. of Physics Letters 10, 105-111 (1997).

[2] R. M. Corless, G. H. Gonnet, D. E. G. Hare, D. J. Jeffrey and D. E. Knuth, "On the Lambert $W$ function", Advances in Computational Mathematics, 5, 329-359 (1996)

[3] S. R. Valluri, D. J. Jeffrey and R. M. Corless, "Some applications of the Lambert $W$ function to Physics", Canad. J. Phys. 78, 823-831 (2000)

[4] K. Roberts and S. R. Valluri, "The Lambert $W$ function, Laguerre Polynomials, and the Zeros of the QCD Partition Function", arXiv:1307.1017v1

[5] N. J. Higham, Functions of Matrices: Theory and Computation, SIAM, Philadelphia (2008)

[6] R. M. Corless, Hui Ding, N. J. Higham and D. J. Jeffrey, "The Solution of $S \exp (S)=A$ is Not Always the Lambert $W$ Function of $A$ ", MIMS EPrint 2007.90, The University of Manchester, 2007

[7] W.-H. Steeb, "Triple Spin Interaction and Entanglement", Z. Naturforsch. 68a, 172-177 (2013)

[8] M. A. Nielsen and I. L. Chuang, Quantum Computation and Quantum Information, Cambridge University Press, Cambridge (2000)

[9] W.-H. Steeb and Y. Hardy, Problems and Solutions in Quantum Computing and Quantum Information, third edition, World Scientific Publishing (2011)

[10] W.-H. Steeb and Y. Hardy, Matrix Calculus and Kronecker Product, second edition, World Scientific, Singapore (2011)

[11] W.-H. Steeb, I. Tanski and Y. Hardy, Problems and Solutions for Groups, Lie Groups, Lie Algebras with Applications, World Scientific, Singapore (2012) 